\title{
Relative Localization of Wireless Sensor Nodes by Using the RSSI Data
}

\author{
Türker TÜRKORAL, Suat YETIŞ \\ Mechatronics Engineering Dept., Dokuz Eylül University \\ Buca, 35390, İzmir (TURKEY) \\ E-mail: turkerturkoral@hotmail.com,suat1479@gmail.com \\ Özgür TAMER, Enes İNANÇ \\ Electrical and Electronics Engineering Dept., Dokuz Eylül University \\ Buca, 35390, İzmir (TURKEY) \\ E-mail: ozgur.tamer@deu.edu.tr, enes.inanc93@gmail.com \\ Levent ÇETIN \\ Mechatronics Engineering Dept., İzmir Katip Çelebi University \\ Çiğli, 35620, İzmir (TURKEY) \\ E-mail: levent.cetin@ikc.edu.tr
}

Received: July 12, 2017

Accepted: January 1, 2018

Published: March 31, 2018

DOI: 10.5296/npa.v10i1.11533

URL: https:// doi.org/10.5296/npa.v10i1.11533

\begin{abstract}
Wireless sensor network is an emerging research field and a crucial infrastructure for Internet of Things (IoT) applications. Sensor nodes of a wireless network may connect via different wireless technologies like Wi-Fi, Bluetooth, ZigBee or WiMAX. In many applications, like field monitoring or smart buildings, the location of the nodes with respect to a global or relative coordinate system is essential information. The main focus of this work is the localization of wireless sensor nodes, using the Received Signal Strength Indicator (RSSI) metric of the wireless telecommunication infrastructure. We present both simulation and measurement results of the proposed method and compare the results with similar work.
\end{abstract}

Keywords: Wireless Sensor Networks, IoT, Localization, RSSI, Wireless Metrics 


\section{Introduction}

A wireless sensor network (WSN) is a network of many (sometimes thousands) of inexpensive miniature devices, called nodes, capable of computation, communication, and sensing [1]. WSNs have gained worldwide attention in recent years, particularly with the improvement in both small and low power electronic application boards and sensors [2]. WSNs are composed of nodes, which are generally identical low power devices. A WSN node is composed of an application board, including a processor (CPU), a memory, a sensor or sensors and a radio transceiver as shown in Fig. 1. Sometimes this configuration may include a positioning system module, like a GPS for outdoor applications [1].

The location of the WSN nodes depends on the application and structure of the WSN [3]. In cases like seismic data monitoring, WSN nodes are fixed to the ground and their position change is tracked via GPS information [4]. In applications like ecosystem monitoring, the node is fixed and its position is predefined to the system, so all calculations can be made according to this predefined data [5].

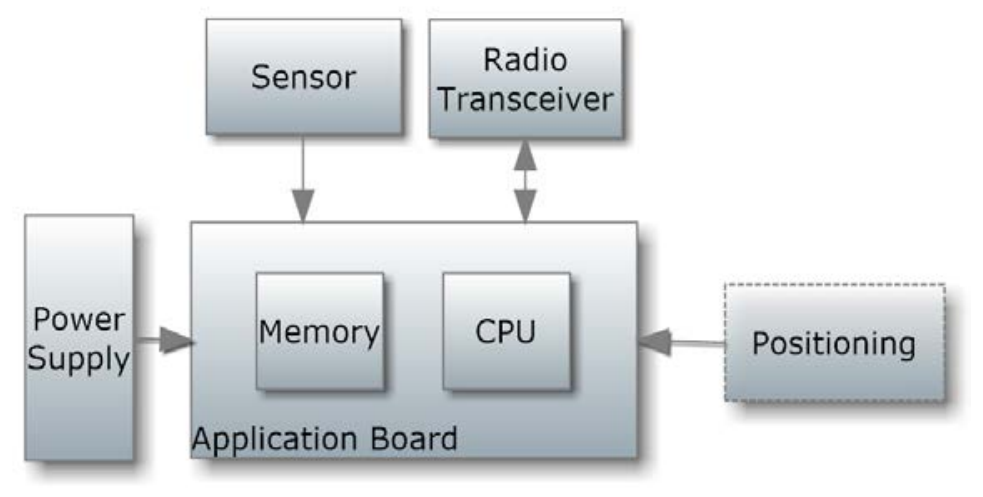

Figure 1. A wireless sensor node structure

Especially in applications with mobile nodes, locating the position of the node is essential, since all the computations will rely on sensor values and the location of the nodes. In outdoor applications, employing a satellite based outdoor positioning equipment like a GPS module or base station signals can be used for positioning the node [6]. However, indoor applications lack of GPS access since satellites cannot cover indoor locations.

In this paper, we propose an RSSI based localization methodology for wireless sensor nodes without any prior information about their absolute positions. A relative coordinate system is formed and nodes are placed according to the estimated positions by the algorithm. If the relation between the actual coordinates and the relative coordinates could be formed the actual positions of the nodes could also be estimated. The rest of the paper is organized as follows: A brief literature survey is presented in section 2. In section 3, we present a brief overview of theoretical background and the methodology. In section 4, we present both the simulation and measurement results and conclude these results in section 5. 


\section{Related Work}

Several approaches have been proposed for the solution of the indoor localization problem. Most widely used classification for localization algorithms of WSNs are range based and range free algorithms [3]. Range-based algorithms employ distance or angle estimates based on measurements of wireless parameters or metrics, while range-free algorithms use connectivity information between nodes to be positioned and static wireless nodes (landmarks) [7]. In this work a range based algorithm is proposed.

Mitilineos et. al. present experimental results for 3 range-based indoor localization schemes of which they employed for their work. The first method is called Cricket and is based on the wireless nodes called crickets. The nodes propagate a periodical beacon signal in the form of an RF pulse and listen to the other nodes beacons. The distance between two nodes is estimated by calculating the Time of Arrival (ToA) of the beacon signal. The localization is then performed according to the relative distances between the nodes by employing trilateration. The second method uses ultra-wideband (UWB) transmitting tags and UWB receivers. UWB receivers are capable of estimating the distance of the UWB transmitter by using the ToA information and the Direction of Arrival (DoA) information of the impinging UWB signal. Location of the UWB is then estimated by employing trilateration based on the ToA information and the DoA. The third approach, called the WAX localization, works on a ZigBee network. First an RSSI fingerprint database for each environment is created based on the offline RSSI measurements. Then the ZigBee RSSI measurements of the nodes are evaluated and trilateration is used to locate the position of the node. Authors claim that among all three methods, WAX is the most accurate one [8]. We propose an RSSI metric based algorithm but the RSSI metric is acquired from the Wi-Fi module instead of ZigBee.

Sivakumar et. al., presented an evolutionary range free approach, to minimize the error in indoor positioning algorithms. The proposed method uses mobile anchors that broadcast their locations as beacon packets. Sensor nodes use this beacon signals to locate their positions. They employ evolutionary algorithms to improve the accuracy of their location estimation [9]. In our work, we do not employ any mobile anchors, the coordinate system is therefore relative. Another range free algorithm is proposed by Chen et. al. The algorithm is based on the RSSI fingerprint database. The indoor environment is divided into regions according to this information. Authors then apply K-nearest neighbour algorithm to estimate the relative location of each node [10]. A fingerprint information based on the measurements is also used in the algorithm presented in this paper.

A self-organizing localization algorithm is proposed by Neuwinger et. al. In their work all nodes are mobile, while the position of the unknown node is assumed as stable during the position estimation operation. The unknown node receives three coordinate information packets of the reference mobile node, and then calculates its coordinates by trilateration. Their results show that error increases with the increasing distance [11]. For the proposed algorithm in this work, no additional package for positioning is needed. An RSSI-based localization scheme considering the trend of the RSSI values obtained from node beacons is proposed by Sahu et. al. Instead of using absolute RSSI values, they use trajectories that are evaluated by applying polynomial modelling, to locate the maximum RSSI value. Authors claim that the 
method is favourable in dynamic domains. Simulation results of the methods show that error ratio reduces with the number of beacons [12]. The study presented in this paper does not need any nodes broadcasting beacon signals, since the coordinate system is relative.

Garcia et al. Proposed two methods where wireless sensors could locate their position using Wi Fi technology. The WSN must be installed on a floor of a building for their study. They propose adopting heuristic training measurement system and adding some fixed access point in the triangular model so that the localization algorithm can adapt to harsh environment of multipath, high temperature, and multibarrier [13]. According to their results, the triangulation system gives more accurate results however, for environments with different type of wall losses performance of the heuristic system is preferable. A hybrid localization system utilizing deductive and inductive methods for wireless sensors inside a floor of a building using WLAN for communication has been proposed by Lloret et al [14]. The authors aim to combine the advantages of the methods to provide more accurate localization results in harsh environments with few base stations and/or few trained points. Unlike the hybrid method proposed by authors, our method does not employ any base stations since it based on a relative coordinate system.

\section{Methodology}

The method is based on the received signal strength information between the nodes. This parameter is evaluated by acquiring the Received Signal Strength Indicator (RSSI) metric from the hardware and processing it. RSSI is defined as the power level indicator of the impinging signal to the receiver node. Hardware manufacturers define generic values for the RSSI information ranging between 0 and 255, while ' 0 ' representing the best signal condition.

RSSI metric is a relative power indicator and we need to process it to evaluate the absolute power. Manufacturers usually share the RF power in $\mathrm{dBm}$ with the RSSI metric. In this work, a Wi-Fi module (F23BUUM13-W2, FN-LINK) with a Realtek RTL8723BU chip is used [15].

The received power depends on the transmitted power and losses. We have the transmitted power information provided by the hardware vendors, so the only parameter affecting the received power is the losses. Losses may arise from several reasons like cable and connector losses, impedance mismatch losses, polarization losses and propagation losses. In our study identical and matched antennas are used which lets us neglect polarization and impedance mismatch losses. Antennas are connected directly to the board, so that we can also neglect cable and connector losses. Therefore, the only cause for the losses is limited to the propagation loss. Propagation loss is generally estimated by using propagation models depending on the characteristic of the environment. Propagation models rely on several environmental parameters including the distance. In this work, we will be using ITU Indoor propagation model, Two-Ray propagation model, and the empirically derived Power-Distance Function propagation model. 


\section{Macrothink}

\subsection{Positioning Algorithm}

For the simulation, 4 WSN nodes are generated by using MATLAB ${ }^{\odot}$. Initially, the only information available is the estimated distance between the nodes. The simulation procedure uses the estimated distance data to locate the actual positions of the nodes. The localization method requires no initial position information. The reference locations are neither fixed nor initially known so the estimated location is a relative location with respect to the reference locations. The first reference location is the first node, node 'a' which will be placed at exactly the origin $(0,0)$ of the relative Cartesian coordinate system as presented in Fig. 2.
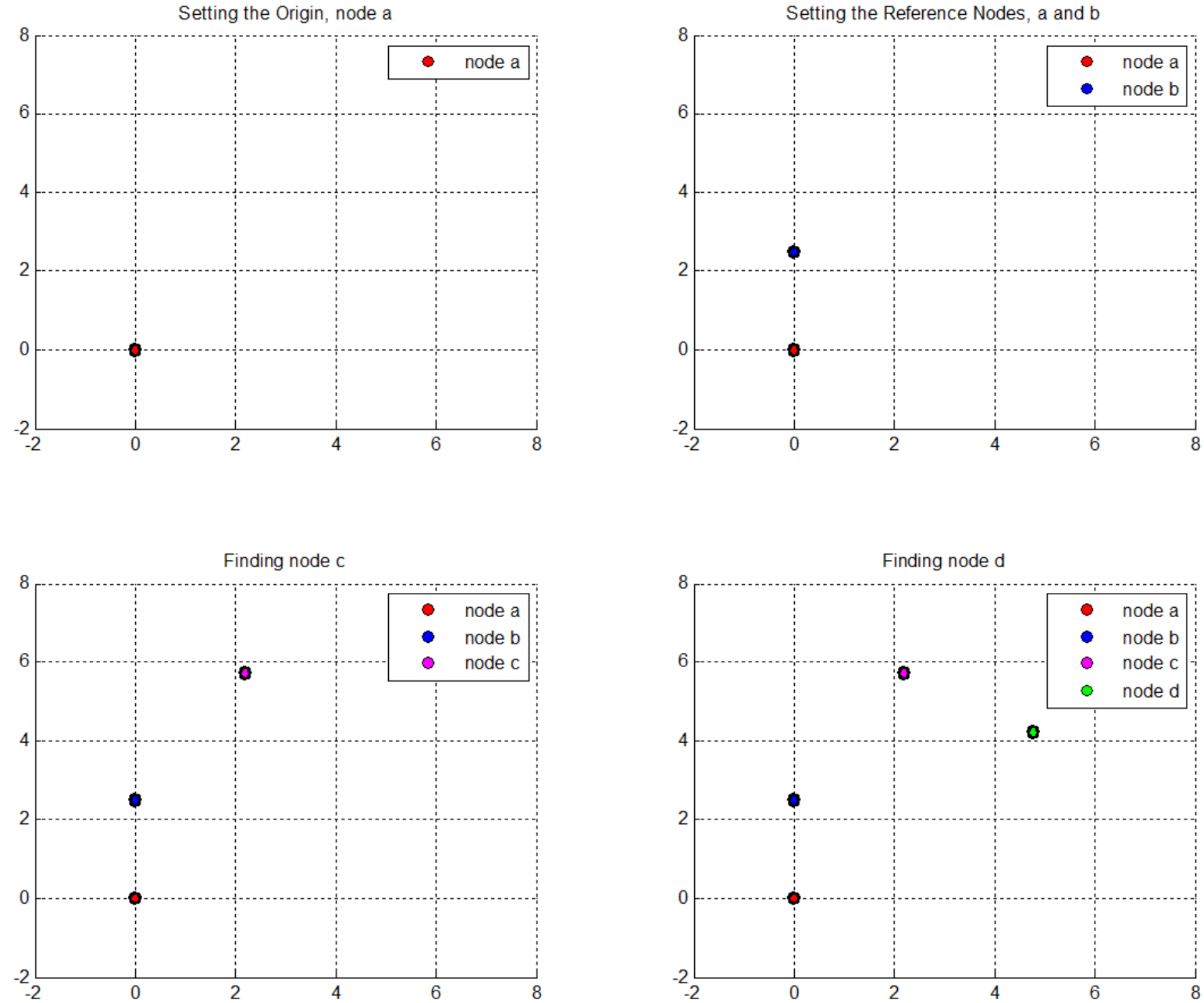

Figure 2. Creating the relative coordinate system

After creating the origin of the coordinate system, we continue with forming the $\mathrm{x}$ and $\mathrm{y}$ axes by using the second node ' $b$ '. Since we have a distance estimation of node ' $b$ ' as $L_{a b}$, and only the origin of the coordinate system, we can place the node at any point, for simplicity, we place it on the ' $y$ ' axis as can be seen in Fig. 2. By locating the node ' $b$ ' at the point $\left(0, L_{a b}\right)$ and forming the $\mathrm{y}$-axis, we also form the $\mathrm{x}$-axis which is also perpendicular to it and both are starting from the origin, namely node ' $a$ '. Based on these locations a relative coordinate system is generated.

Following the formation of the relative coordinate system, we may start locate the other 


\section{Macrothink}

nodes, ' $c$ ' and ' $d$ ' on the relative coordinate system, with the use of the estimated distances in between all the nodes. To locate a node with the estimated distances between the nodes, trilateration method is used. Trilateration is a widely used method for estimating the location by using at least three reference points $[16,17,18]$. Distance with only a single reference point gives a circular possible position cluster and the radius equals to the distance one node from another. However, the addition of the location of the $2^{\text {nd }}$ node, trilateration gives a more precise result, 2 possible locations of the third node, because the circles intersect only twice. The third node reduces the possible location to 1 and lets us to estimate the location of the node in a two dimensional coordinate system. More reference points improve the accuracy and result in more precise location estimation.

In our work, to estimate the location of the third node we still have two intersection points and we have the opportunity to select one of them. Since we are using a relative coordinate system depending on the references and both intersections are just mirrored versions of each other. Our selection is the one with a positive $x$ value for node 'c' without losing generality.

Location of the $4^{\text {th }}$ node is also estimated by using the distance to ' $a$ ' and ' $b$ ' nodes and choosing one of the two intersection points. But this time we also know its distance with node ' $c$ ' and we choose the intersection closest to this distance. A block diagram of the algorithm is presented in Fig 3.

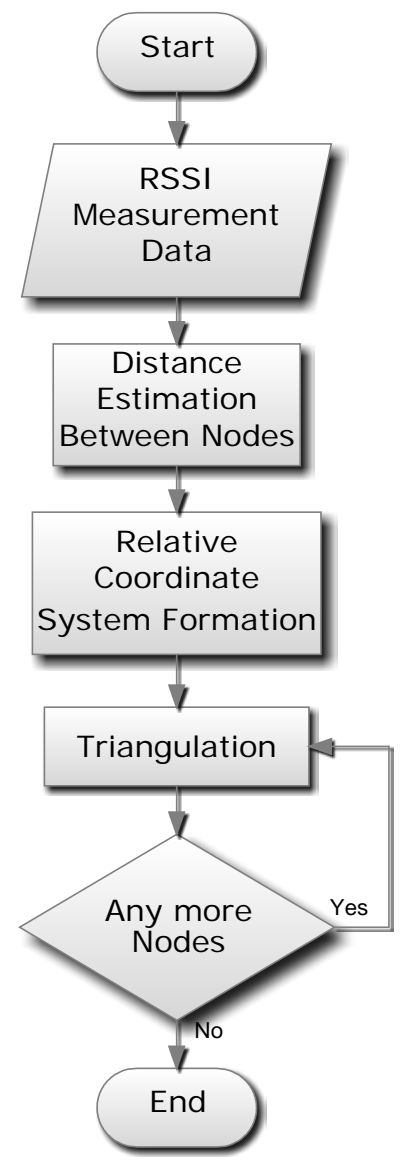

Figure 3. Flow chart of the algorithm 


\subsection{Distance Estimation Based on Propagation Models}

By using the RSSI data, it is possible to estimate the propagation loss between two nodes, as mentioned above. However, the relation of the distance and propagation loss depends on the environment and the path loss model employed. Since the RSSI data varies rapidly an average value of 20 measurements is used as the RSSI measurement. In this work distance is estimated based on four different propagation models presented below.

\subsubsection{ITU Indoor Path Loss Model}

ITU indoor path loss model is recommended by the International Telecommunication Union under recommendation number P.1238-8 and it is widely recognized by the researchers [19]. Propagation loss based on the ITU model can be estimated using (Eq. 1).

$$
L_{i t u}=20 \log _{10}(f)+N \log _{10}(d)+L_{f}(n)-28 d B
$$

In the equation, $L_{i t u}$ is the total power loss, $f$ is the operating frequency, $d$ is the distance between the source and the receiver, $N$ is the Power Loss Coefficient and $L_{f}(n)$ is the Floor Penetration Loss Factor. The Power Loss Coefficient Value $(N)$ and the Floor Penetration Loss Factor $\left(L_{f}(n)\right)$ depend on the environment. Several parameters have been proposed by researchers for different environments [20,21]. We also need to estimate the best suiting parameters for our communication environment [21]. When these values are placed in the equation, the distance will only depend on the RSSI value.

The model is affected by the floors of which the receiver and transmitter are located on [21]. Nodes can be located on different floors and the parameter $L_{f}(n)$ changes accordingly [21]. However, in this study, all measurements are taken on the same floor, so the $L_{f}(n)$ is presumed as a constant value.

Total loss, $L_{t}$, is to be found with the difference from the transmitted signal power and the received signal power. Since the receiver and the transmitter nodes are on the same floor and the transmitted signal power is constant $(13 \mathrm{dBm}$, [15]), the difference with the received power gives the total loss, $L_{t}$. Adding the other parameters of the model gives the resulting power-distance relation:

$$
P_{t}-R S S I=20 \log _{10}\left(2.4 \times 10^{9}\right)+N \log _{10}(d)+L_{f}(0)-28 d B
$$

In Eq. 2, $N$ and $L_{f}(n)$ parameters vary by the frequency and the floor material. Since the nodes are on the same floor, the floor difference $n$ equals to ' 0 '. To evaluate the optimum value for the $N$ and $L_{f}$ coefficients, the distance value corresponding to each possible combination is compared with the actually observed distance. As can be observed in Fig. 4, $N$ and $L_{f}$ values providing the least mean distance difference with the fingerprint data occur as $\mathrm{N}=30$ and $\mathrm{L}_{\mathrm{f}}=11$. The coefficients are consistent with the information in the literature [21]. 


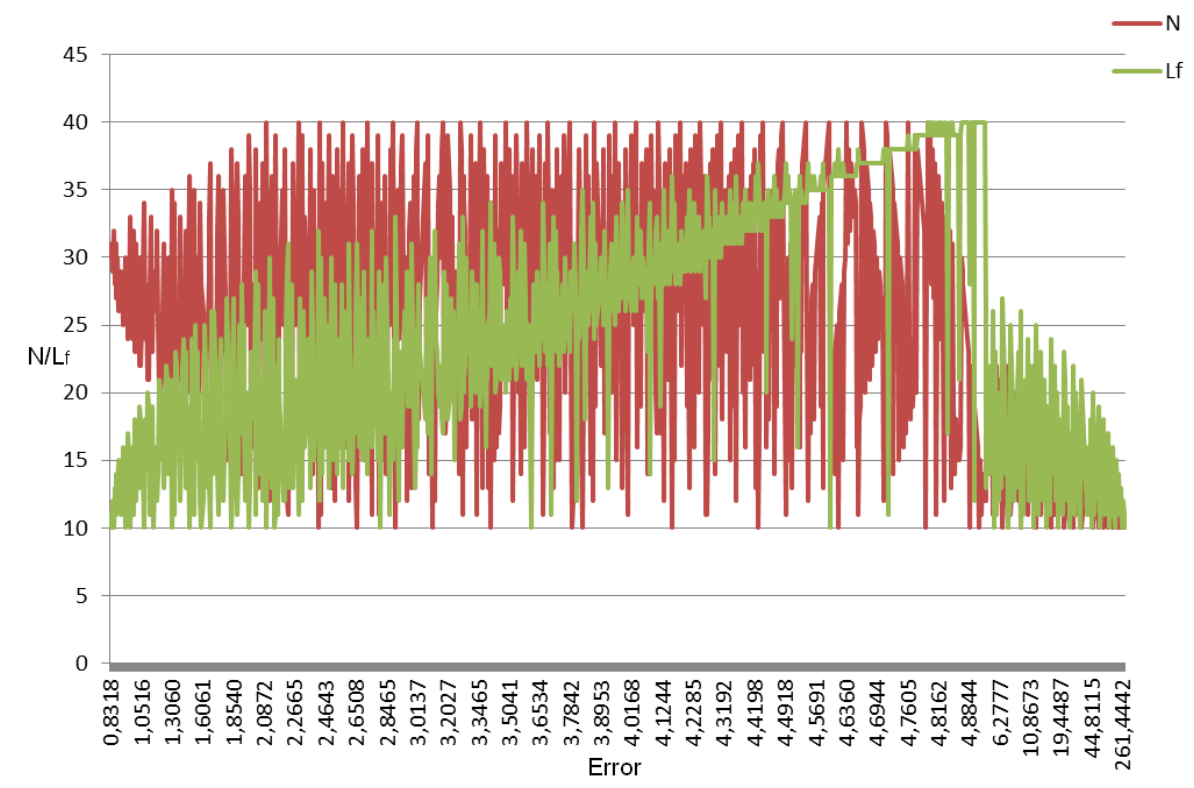

Figure 4. Error Performance of the ITU Model for $N$ and $L_{f}$ coefficients

\subsubsection{Free Space Propagation Model}

Free Space Propagation Model is the most basic propagation model and does not take in any reflections or refractions into consideration [22,23]. Received power in this model can be evaluated as;

$$
P_{\text {RSSI }}=P_{t}+G_{r}+G_{t}-L_{f s}
$$

Where $L_{f s}$ is the free space loss and given as;

$$
\mathrm{L}_{\mathrm{fs}}=20 \log \left(\frac{4 \pi R}{\lambda}\right)
$$

In this method, the received power $P_{R S S I}$ is obtained from the RSSI metric, while the transmitted power $P_{t}$ is given as $13 \mathrm{dBm}$ as given in the datasheet of the hardware [15]. The frequency is $2.4 \mathrm{GHz}$ hence the wavelength $(\lambda)$ is found $12.5 \mathrm{~cm}$. The modules and the antennas that are used are identical so $G_{r}$ and $G_{t}$ values are also even and equal to $1.7 \mathrm{~dB}$ for that frequency [24].

\subsubsection{Two-Ray Ground Reflection Model}

Two-Ray Ground Reflection Model is similar to Free Space Propagation Model, but it also includes a reflected wave from the nearest reflecting surface (Eq. 8) [22].

$$
P_{\text {RSSI }}=P_{t}+G_{r}+G_{t}-L_{t r}
$$

In this model, $L_{t r}$ represents the loss including both the ground reflected wave and the 
line of sight wave (See Eq. 9).

$$
L_{t r}=20 \log \left(\frac{d^{2}}{h_{t} h_{r}}\right)
$$

In Fig. 5 the two-ray propagation model is presented including the transmitter and the receiver antennas, and the reflected and line of sight rays. The heights of the transmitter and the receiver antennas are equal in this work, and $h$ is very short when compared to $d$. So the equation becomes as presented in (Eq. 7).

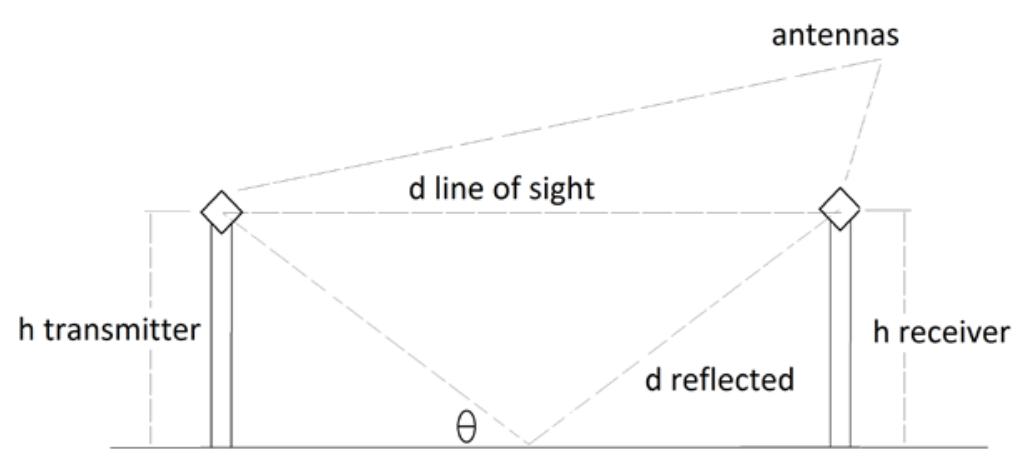

Figure 5. Line of sight and reflection rays between the antennas

$$
P_{R S S I}=P_{t}+G_{r}+G_{t}-40 \log \left(\frac{d}{h}\right)
$$

\subsubsection{Power-Distance Function}

Power-Distance Function is a function derived from the experimental fingerprint data evaluated during this study. Experimental results are fitted to an exponential function by using MATLAB ${ }^{\odot}$ software, and the coefficients of the function are determined. The actual Power-Distance Function is presented in Eq. 8.

$$
d=a e^{-b \cdot P_{R S S I}}
$$

Experimental results and the derived function are presented in Fig. 6. According to the measurements a and b values are evaluated as 0,09878 and 0,06658 respectively. Note that these parameters are for the test bed environment only. 


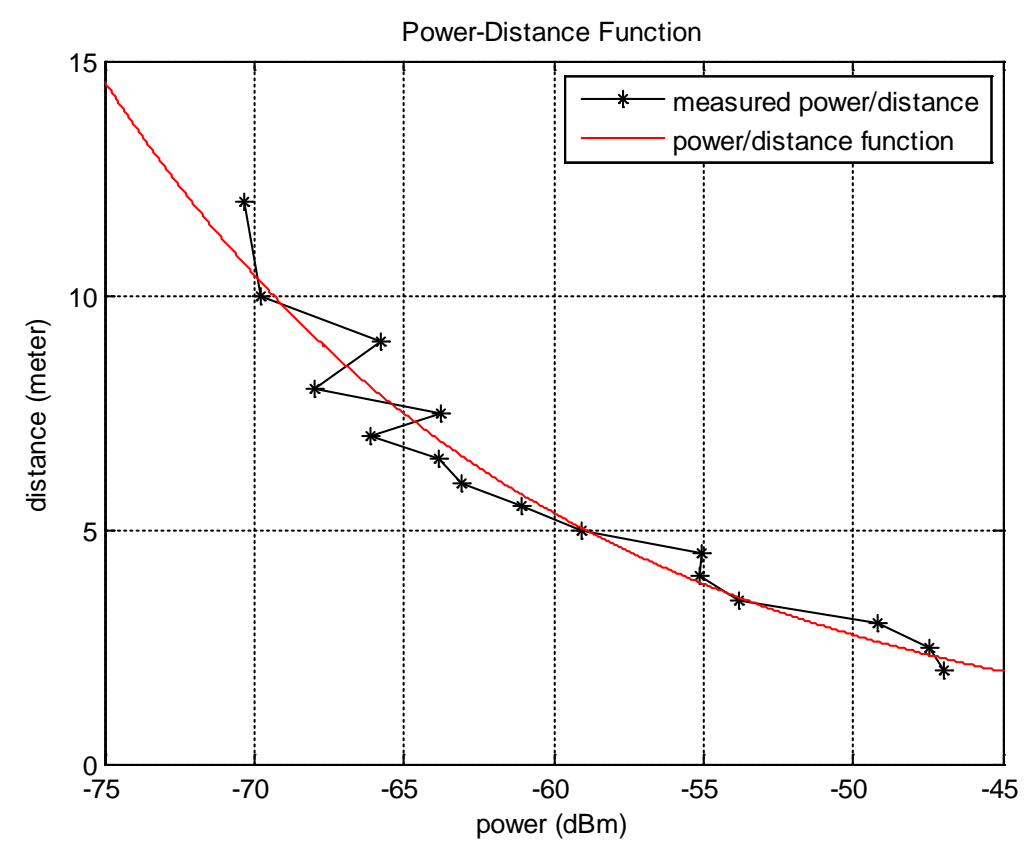

Figure 6. Power-Distance Function

\section{Results}

According to the methodology presented above; first, MATLAB ${ }^{\circledR}$ simulation results are evaluated, then WSN's are placed on a testbed and several measurements have been acquired. Results of these studies are presented below.

\subsection{Simulation Results}

The simulation process of the system is carried out through MATLAB ${ }^{\complement}$ software. Four WSN nodes are defined initially. The locations of the nodes are predefined to the system. Fig. 7 presents the location of each node in Cartesian coordinate system. The distance between each node is evaluated according to the predefined locations of the nodes (Table 1).

Table 1. Node coordinates and the distances in between

\begin{tabular}{|l|l|}
\hline $\mathbf{L}_{a \mathbf{b}}$ & $2,50 \mathrm{~m}$ \\
\hline $\mathbf{L}_{\mathbf{a c}}$ & $6,11 \mathrm{~m}$ \\
\hline $\mathbf{L}_{\mathbf{b c}}$ & $3,88 \mathrm{~m}$ \\
\hline $\mathbf{L}_{\mathbf{a d}}$ & $6,39 \mathrm{~m}$ \\
\hline $\mathbf{L}_{\mathbf{b d}}$ & $5,09 \mathrm{~m}$ \\
\hline $\mathbf{L}_{c d}$ & $3,00 \mathrm{~m}$ \\
\hline
\end{tabular}

\begin{tabular}{|c|c|c|}
\cline { 2 - 3 } \multicolumn{1}{c|}{} & $\mathbf{x}$ & $\mathbf{y}$ \\
\hline $\mathbf{a}$ & 0 & 0 \\
\hline $\mathbf{b}$ & 0 & 2,5 \\
\hline $\mathbf{c}$ & 2,2 & 5,7 \\
\hline $\mathbf{d}$ & 4,8 & 4,2 \\
\hline
\end{tabular}

Additive White Gaussian Noise (AWGN) is added to the simulated RSSI value. A number of measurements with AWGN are simulated. The number of the simulated data values is called the realization number, ' $r$ num'. 


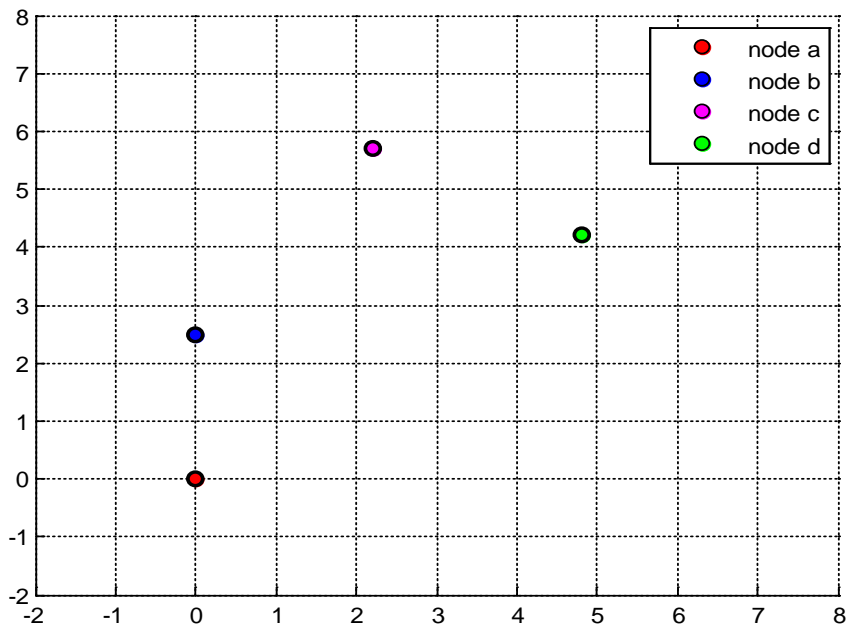

Figure 7. Predefined locations of the WSN nodes a, b, c and d

Distance estimations for node ' $b$ ' are located on the relative $y$-axis (black circles in Fig. 8). Then the mean value of these values is evaluated to have an estimated value for node ' $b$ ' as shown as a yellow spot in Fig. 8.

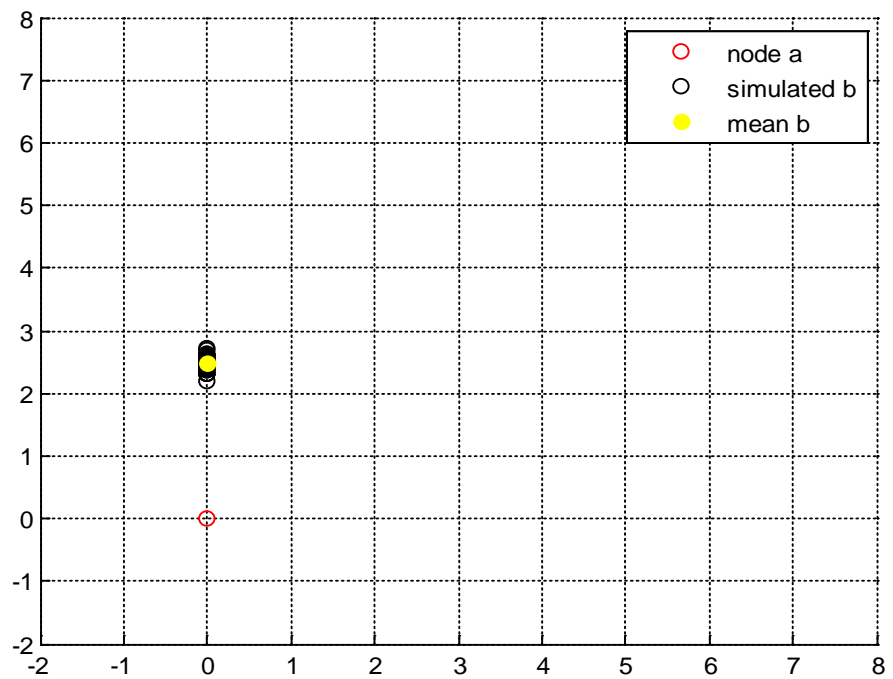

Figure 8. Location of node ' $a$ ' and estimated location of ' $b$ ' by the distance set $L_{a b}$

The position of the $3^{\text {rd }}$ node, ' $c$ ', can be estimated by using the set of distance values $L_{a c}$, $L_{b c}$, and $L_{a b}$ (Fig. 9). 


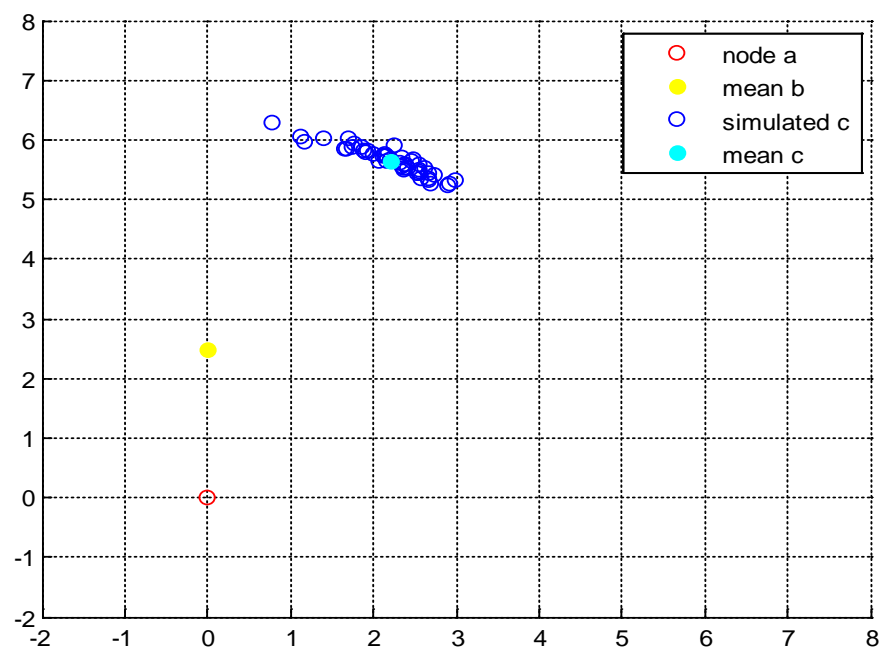

Figure 9. Location of node ' $a$ ' and estimated location of ' $b$ ' by the distance set $L_{a c}$ and $L_{b c}$

The position of node ' $c$ ' is estimated by, calculating the intersection of the two circles and taking the positive one into account, as presented in Fig. 10.

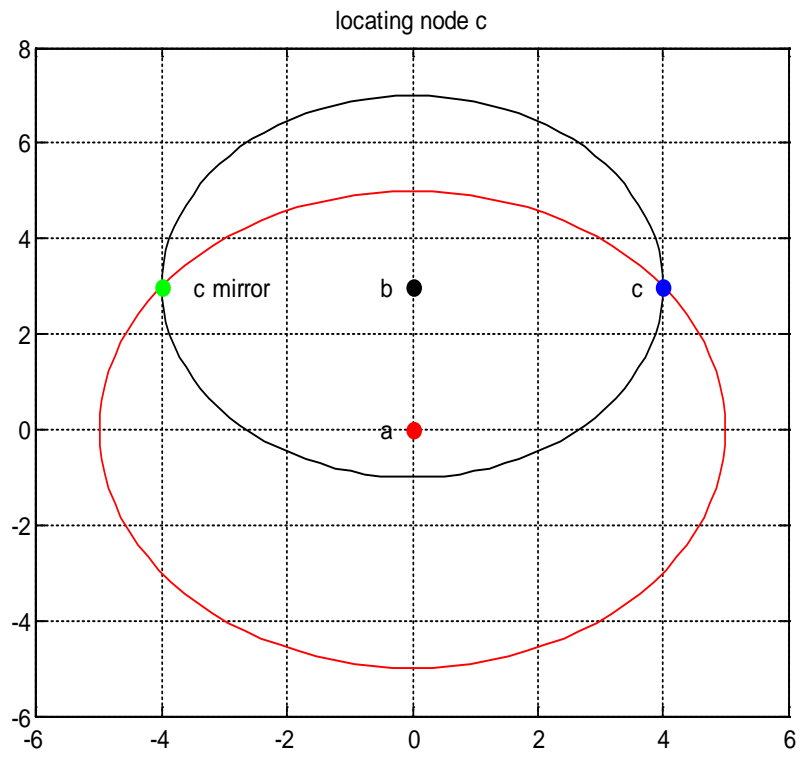

Figure 10. Locating node ' $c$ ' by triangulation

The first circle in Fig. 10 has a centre at node ' $a$ ', with a radius equal to $L_{a c}(n)$ where $n$ is the number of iterations, while the second circle is centred at node ' $b$ ' with a radius of $L_{b c}(n)$. These two circles intersect at two points in space if the centres are not located at the same point and if the $L_{a c}$ value is not greater than the summation of $L_{a b}$ and $L_{b c}$.

By locating node ' $c$ ', we now have 3 of the nodes located on the coordinate system. When we acquire distance information from a $4^{\text {th }}$ node, with respect to the first two nodes, all we need 


\section{Macrothink}

to do is to repeat the previous process that is done for node ' $c$ '. Locations of the calculated node ' $d$ ' positions according to the simulated data are presented with green circles in Fig. 11 while the mean of these locations is shown as a pink spot and it is the estimated location for node ' $d$ '.

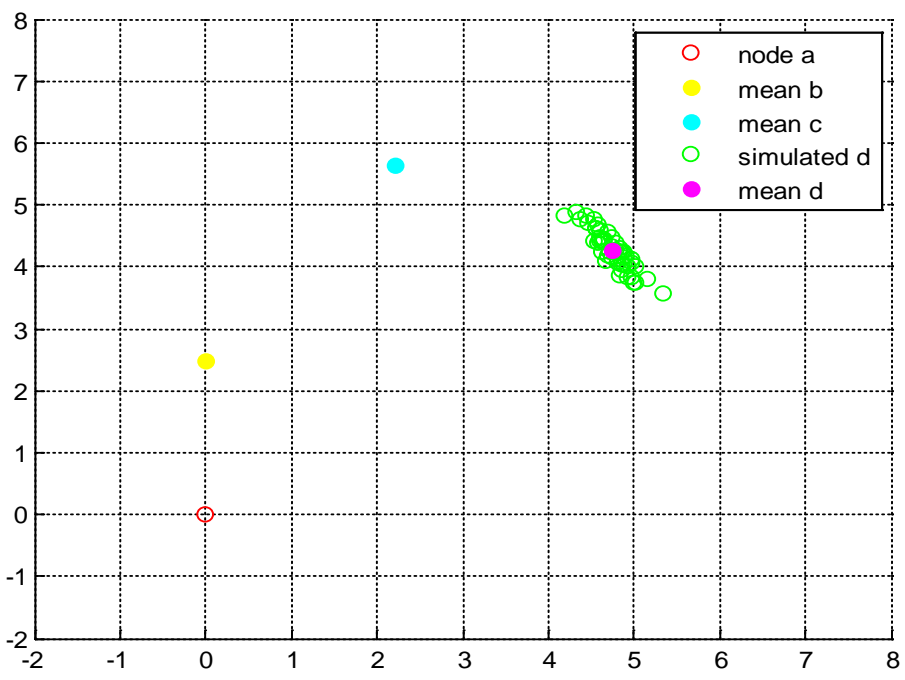

Figure 11. Estimations and the mean value of node ' $d$ ' set by using $L_{a d}$ and $L_{b d}$

By using the distance data between the nodes, ' $r$ num' (realization number) number of noisy simulation data is created and, the locations of nodes on the Cartesian coordinate system are estimated. For the simulation results that are presented in Fig. 12, ' $r_{\text {num }}$ ' and Signal to Noise Ratio (SNR) are chosen 50 and 20 respectively.

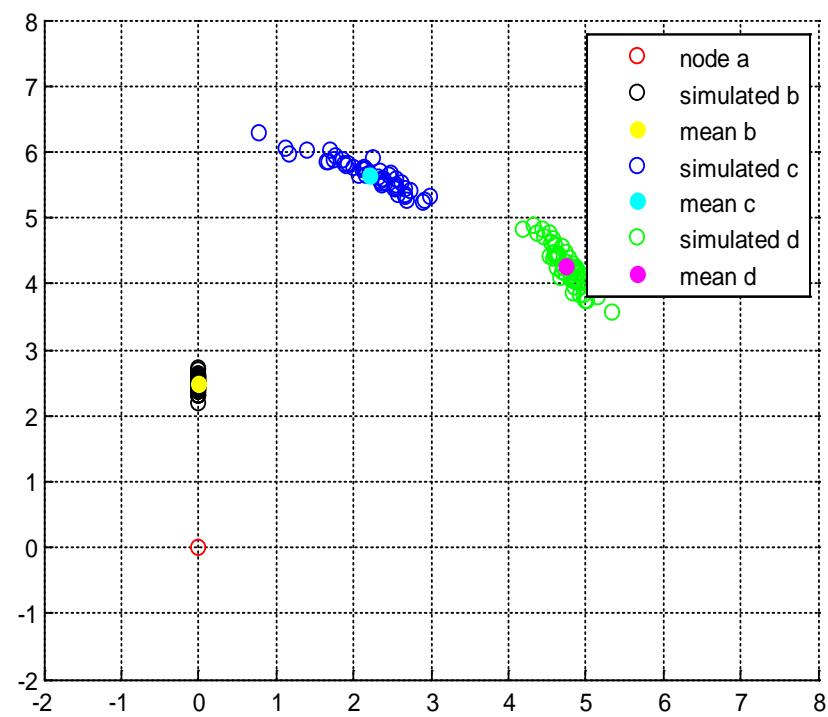

Figure 12. Simulation results for 'rnum' $=50, \mathrm{SNR}=20$

For the simulation with an SNR value of 20 and 50 iterations $\left(r_{\text {num }}=50\right)$, positioning 


\section{Macrothink}

errors with respect to the actual coordinates of nodes ' $b$ ', ' $c$ ' and ' $d$ ' occur as $0.01 \mathrm{~cm}$., 4.67 $\mathrm{cm}$. and $7.89 \mathrm{~cm}$. respectively. No error has been defined for node ' $a$ ' since it is the initial reference point and rest of the estimated positions depends on its position. The error performance is based on the difference between the nodes' actual locations and the mean of simulation results.

In Fig. 13, the simulation results are presented for 10 iterations and an SNR value of 10. The positioning errors for this case occur as $6.82 \mathrm{~cm}$., $49.62 \mathrm{~cm}$. and $36.75 \mathrm{~cm}$. for the nodes ' $b$ ', ' $c$ ' and ' $d$ ' respectively.

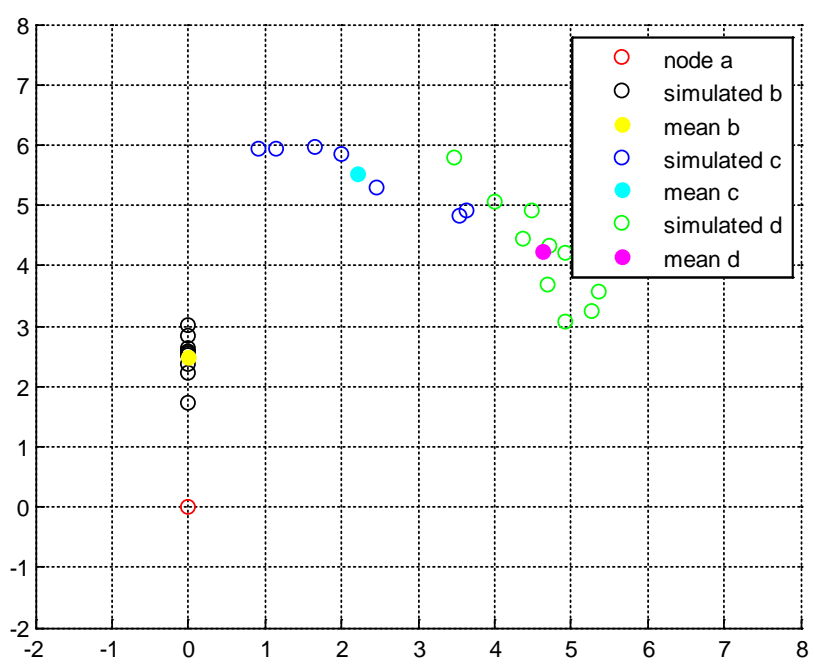

Figure 13. Simulation results for 'rnum' $=10, \mathrm{SNR}=10$

Fig. 14 demonstrates the simulation results when 30 sample values are considered with an SNR value of 5 . The error values of the system under the given conditions for the nodes ' $b$ ', 'c' and ' $d$ ' are $11 \mathrm{~cm} ., 97.53 \mathrm{~cm}$. and $43.86 \mathrm{~cm}$. respectively.

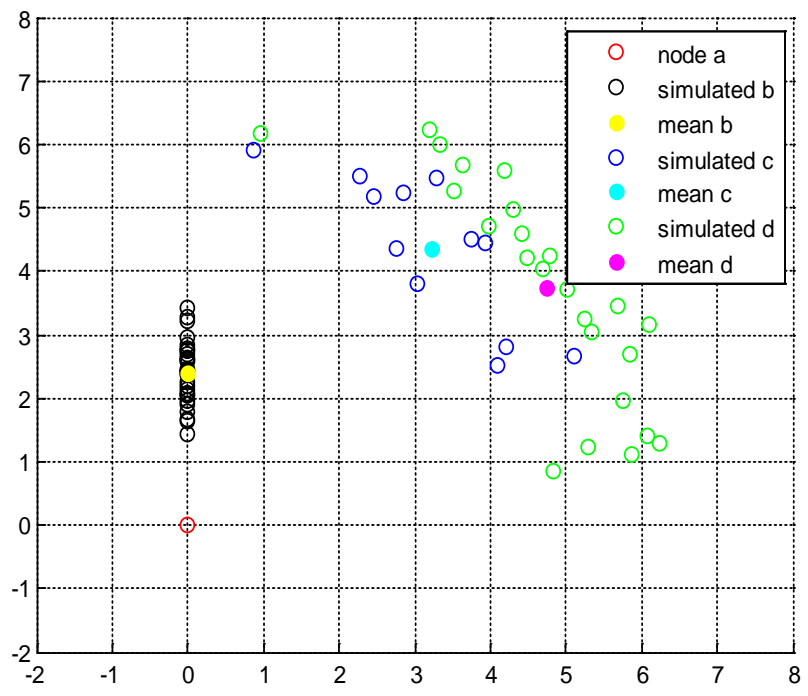

Figure 14. Simulation results for 'rnum'=30, SNR=5 


\subsection{Experimental Results}

In the content of this work, a testbed is constructed in an indoor environment by using communication modules and single board computers forming simple WSN communication nodes. Each WSN node consists of a Raspberry Pi 2 single board computer and a Realtek RTL8723BU USB Wi-Fi module.

These WSN communication nodes are placed at the same positions accordingly with the simulated data. The RSSI metric of the Wi-Fi chip is acquired via the driver on the SBC. Three propagation models and the power function based model are used to estimate the distance values and place the WSN nodes on a relative Cartesian coordinate system.

During the measurement process, Automatic Gain Control (AGC) of the Wi-Fi chip is disabled to acquire a reading that depends only on the distance and the environmental conditions.

For all the power-distance relation methods that are presented to derive the distance values between the nodes, a number of measurements are acquired and the mean of the RSSI values are used to estimate the distance values. All three path loss models and the derived power distance function have been compared, and the results are presented in Fig. 15. In this figure, an offset has been added to both the Free Space Propagation Model and the Two-Ray Propagation Model to make them comply with the measurement results. The offset value for the Free Space case is $24 \mathrm{~dB}$ while for the Two-Ray case it is $7 \mathrm{~dB}$. These offset values are based on the measurement values and may change depending on the environment.

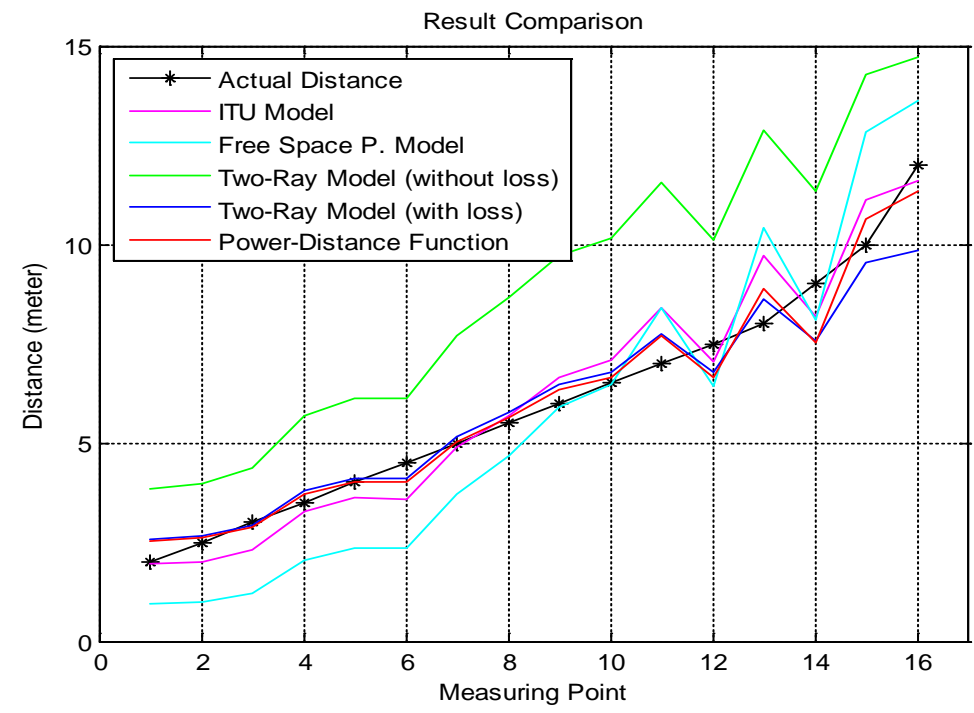

Figure 15. Performance Comparison of the estimations of different propagation models with actual measurement values

The RSSI measurements, recorded in between 2-12 m. are observed and the difference between the actual positions and the ITU model results are compared for all the $N$ and $L_{f}$ values between 10 and 40. Error performance of the ITU model by the constant parameters can be seen in Fig. 4 . The least mean error $(0.8318 \mathrm{~m})$ occurs when $N=30$ and $L_{f}=11$ and for all the distance estimations made with ITU model these values were used. 
We used these parameters to estimate the distance between the WSN nodes. For 4 nodes, there are 6 distance values in between the nodes, that are $L_{a b}, L_{a c}, L_{b c}, L_{a d}, L_{b d}$, and $L_{c d}$. As for the acquirement of the RSSI values, corresponding distances are estimated. Localization results of the nodes based on the procedure which is presented in section 3 is shown in Fig. 16.

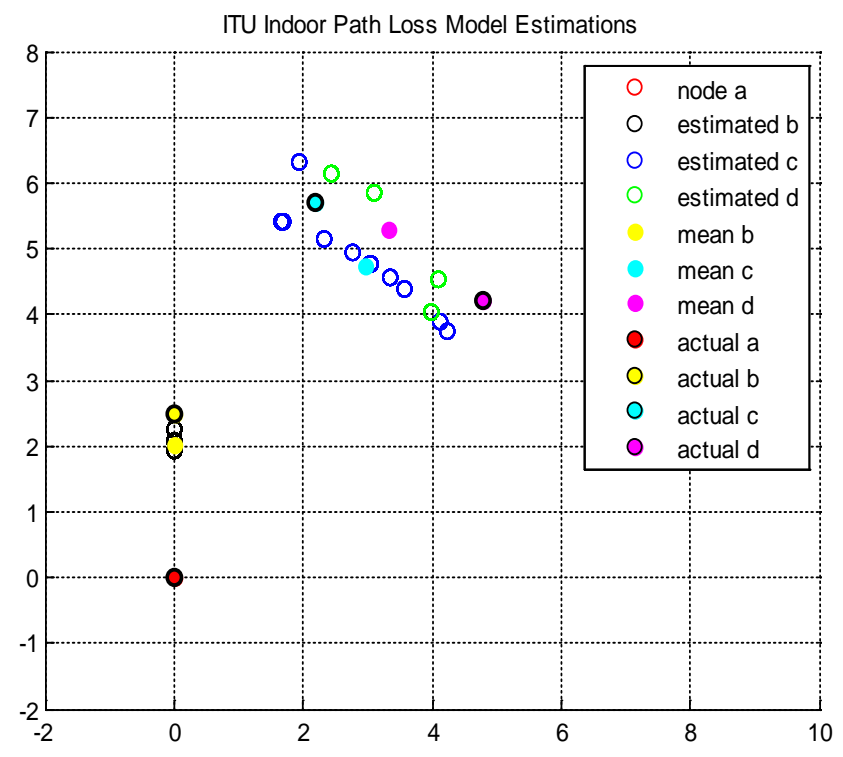

Figure 16. ITU Model experimental results

The estimations exhibit the positioning errors of $49.14 \mathrm{~cm} ., 123.03 \mathrm{~cm}$. and $184.06 \mathrm{~cm}$. for the nodes ' $b$ ', ' $c$ ' and ' $d$ ' respectively. The mean error of the ITU Model is $118.74 \mathrm{~cm}$. and it is similar to the error performance of the simulation results for the same number of iterations when SNR value is approximately 0.2 .

The results of the localization procedure using the Free Space Propagation Model are presented in Fig. 17. 


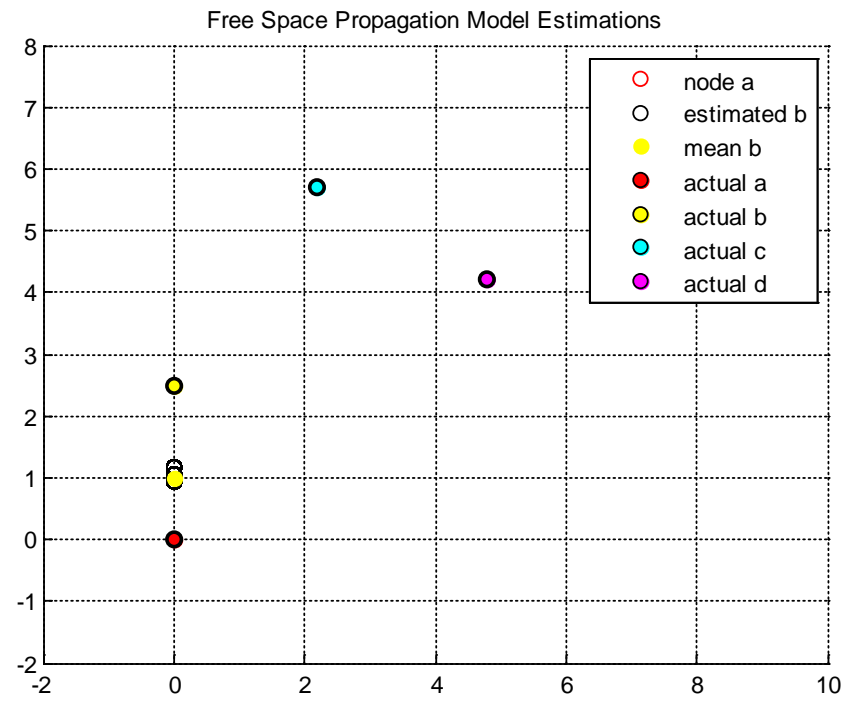

Figure 17. Free Space Propagation Model localization results

Free Space Propagation Model does not seem appropriate to estimate the distance under these conditions. The nodes ' $c$ ' and ' $d$ ' could not be estimated by using the results of Free Space Propagation Model in spite of the fact that an additional offset value of $24 \mathrm{~dB}$ is used in the formula.

Experimental positioning results of the Two-Ray Ground Reflection Model can be seen in Fig. 18. As mentioned in Section 2, an extra power offset value of $7 \mathrm{~dB}$ is used to increase the accuracy of the model.

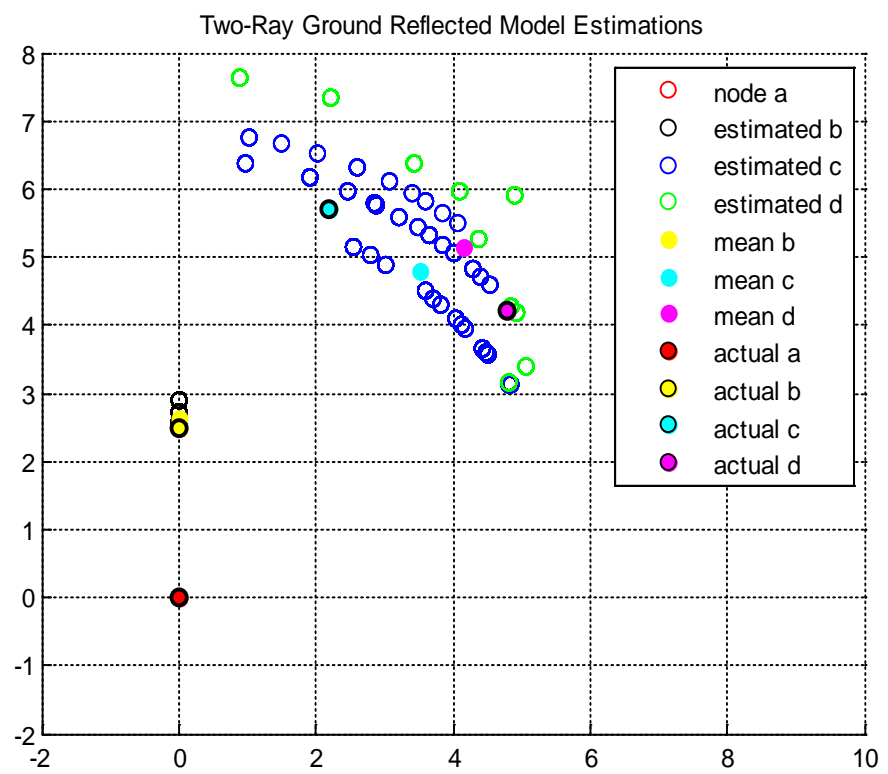

Figure 18. Two-Ray Ground Reflection Model Results

This model exhibits a considerably better error performance than the results of previous 


\section{Macrothink}

models. Two-Ray Ground Reflected Model exhibits a mean positioning error of $96.14 \mathrm{~cm}$. in average and $14.27 \mathrm{~cm} ., 159.72 \mathrm{~cm} ., 114.45 \mathrm{~cm}$. for the nodes ' $b$ ', ' $c$ ' and ' $d$ ' respectively. It is also observed that when the SNR value is approximately 1.5, the mean error performance of Two-Ray model and simulation results seem to be similar.

The last method that is used to estimate the distance is the experimentally derived Power-Distance Function. With the use of this function, the distances between the nodes are estimated by using the mean value of the RSSI measurements. The locations of the nodes are found via the set of these distance values. The results are presented in Fig. 19.

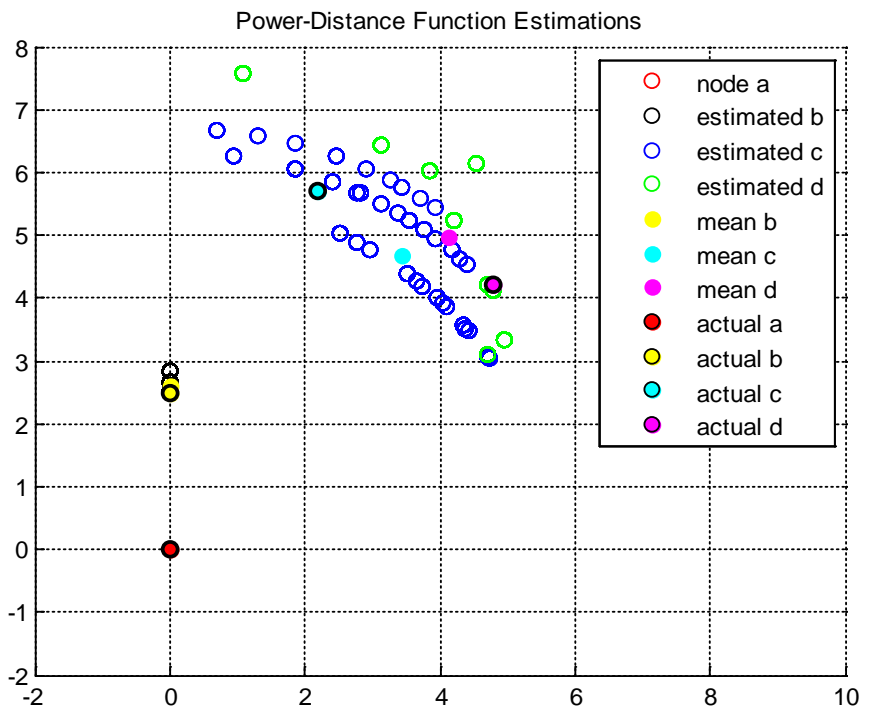

Figure 19. Power-Distance Function Estimations

The error performance of the last method is barely better than the previous models. The mean error value of this method is $90.12 \mathrm{~cm}$. and this result of error is similar to the error performance of the simulation when SNR is approximately 1.9 for the same number of iterations. The mean relative positioning errors of the nodes 'b', 'c' and 'd' are $9.32 \mathrm{~cm}$., $159.18 \mathrm{~cm}$. and $101.85 \mathrm{~cm}$. respectively.

\subsubsection{Comparison of the Results}

The methods of power-distance relation are all observed and compared with the simulation results. All results and the simulation results for SNR=2 can be seen in Fig. 20. 


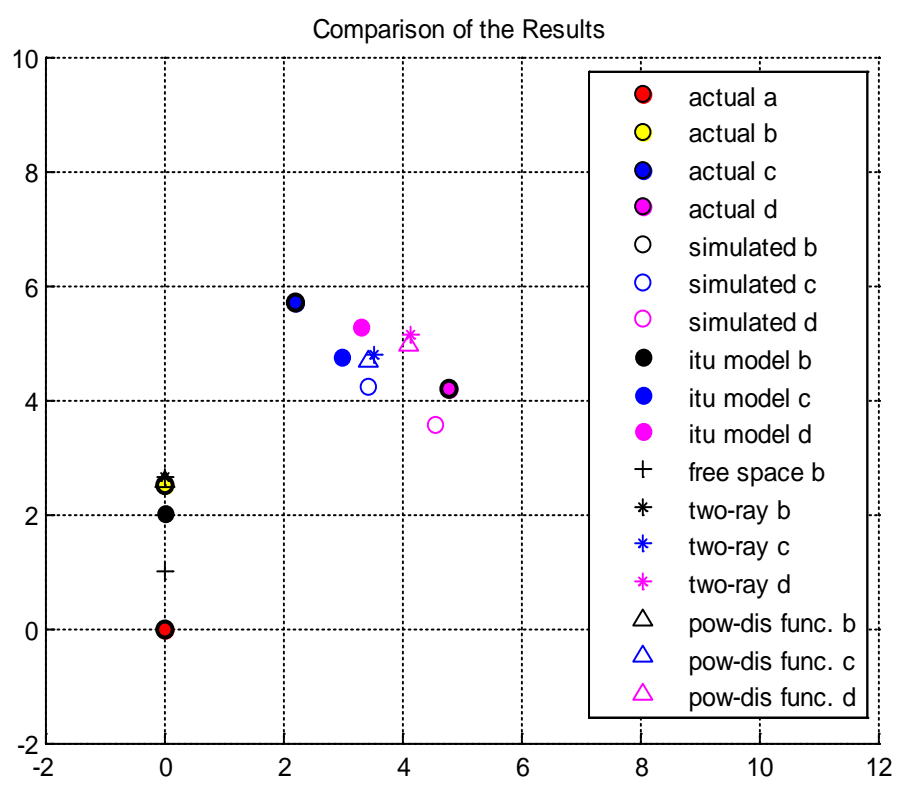

Figure 20. Comparison of the Results

For all the methods that are used, the mean and the individual positioning errors of all the nodes are presented in Table 2. For the simulation process, SNR is 2.

Table 2. Comparison of error values

\begin{tabular}{|l|c|c|c|c|}
\hline Errors (m) & node b & node c & node d & mean \\
\hline simulation & 0,0034 & 1,9151 & 0,6625 & 0,8603 \\
\hline itu model & 0,4914 & 1,2303 & 1,8406 & 1,1874 \\
\hline free space & 1,5170 & - & - & - \\
\hline two-ray & 0,1427 & 1,5972 & 1,1445 & 0,9614 \\
\hline pow-dis func. & 0,0932 & 1,5918 & 1,0185 & 0,9012 \\
\hline
\end{tabular}

As a result, it is observed that for some positions, different models yield better results, but overall, the best result is given by the Power-Distance Function. Additionally, Two-Ray Ground Reflection Model results are very close to the Power-Distance Function.

\section{Conclusion}

In this work, the locations of 4 WSNs are estimated by using both the simulation data and the experimental results. The four propagation models; ITU Indoor Propagation Model, Free Space Propagation Model, Two-Ray Propagation Model and experimentally derived Power-Distance Function are used to estimate the distance between the nodes. By using these distance values, every node is set on a map and located on the relative Cartesian coordinate system by using the proposed localization algorithm. The results and the error performances of the corresponding propagation models are observed and compared with the simulation results and alternative propagation models covered in this paper. It is observed that different 
propagation models yield different results for different distance ranges. At distances close to the limits of the test bench, best results are observed with the Power-distance Function, as well as Two-Ray Ground Reflection Model; but for the midrange distances, ITU Indoor Path Loss Model presents the best outcomes.

It is considered to employ all of the models except the Free Space model for estimating the distance between nodes, and weight the outputs according to the region of the receiver and related environmental conditions to improve the performance.

Also the Wi-Fi operating frequency is $2.4 \mathrm{GHz}$, which is a very common wireless communication frequency and there esixt many interfering devices at this frequency band. Operating the system at $5 \mathrm{GHz}$ frequency band interference will be reduced and the performance of the system will increase. Other Wi Fi metrics, like ToA and TDoA will also be employed for triangulation as a future work and the results will be compared. According to the results, an adaptive algorithm weighting the localization results of different metrics can also be employed.

\section{Acknowledgement}

This work is supported by The Scientific and Technological Research Council of Turkey (TÜBİTAK) under grant no. 114E659.

\section{References}

[1] Akyildiz, I. F., Su, W., Sankarasubramaniam, Y., \& Cayirci, E. (2002). "Wireless sensor networks: a survey.” Computer networks, 38(4), 393-422. https://doi.org/10.1016/S1389-1286 (01)00302-4

[2] Prabhu, B., Balakumar, N., \& Antony, A. J. (2017). "Wireless Sensor Network Based Smart Environment Applications." International Journal of Innovative Research in Technology - IJIRT, Volume 3, Issue 8, Jan. 2017.

[3] Han, G., Jiang, J., Zhang, C., Duong, T. Q., Guizani, M., \& Karagiannidis, G. K. (2016). "A survey on mobile anchor node assisted localization in wireless sensor networks". IEEE Communications Surveys \& Tutorials, 18(3), 2220-2243. https://doi.org/10.1109/COMST. 2016.2544751

[4] Yamabe, Y., Sasaki, H., Inatome, T., \& Tani, A. (2014). Fundamental Test of Seismic Information and Building Damage Data Gathering System using OSHW with Wireless Sensor Network" Computing in Civil and Building Engineering (2014) (pp. 1158-1165). https://dx.doi.org/10.1061/9780784413616.144

[5] Mukhopadhyay, S. C., \& Jiang, J. A. (Eds.). (2013).”Wireless sensor networks and ecological monitoring”(Vol. 3). Springer Science \& Business Media.

[6] Yick, J., Mukherjee, B., \& Ghosal, D. (2008). "Wireless sensor network survey.” Computer networks, 52(12), 2292-2330. https://doi.org/10.1016/j.comnet. 2008.04.002

[7] Pal, A. (2010). “Localization algorithms in wireless sensor networks: Current approaches 
and future challenges.” Network Protocols and Algorithms, 2(1), 45-73. https://doi.org/10. 5296/ npa.v2i1.279

[8] Mitilineos, S.A., Kyriazanos, D.M., Segou, O.E., Goufas, J.N. and Thomopoulos, S.C.A. (2010) “Indoor Localisation With Wireless Sensor Networks”, Progress In Electromagnetics Research, 109, pp. 441-474. http://dx.doi.org/10.2528/PIER10062801

[9] Sivakumar, S., Venkatesan, R. and Karthiga, M. (2015) "Evolutionary Approaches For Minimising Error In Localisation Of Wireless Sensor Networks”, International Journal of Sensor Networks, 17(1), p. 17. http://dx.doi.org/10.1504/ijsnet.2015.067587.

[10] Chen, Y., Liu, Z., Li, Y. and Wang, Y. (2014) “Indoor localization algorithm for wireless sensor network based on range-free", in Communications in Computer and Information Science. Springer Science + Business Media, pp. 221-230. https://doi.org/10.1007/978-3642- 54522-1_22

[11] Neuwinger, B., Witkowski, U., \& Rückert, U. "Ad-Hoc Communication And Localization System For Mobile Robots.” FIRA RoboWorld Congress (pp. 220-229). Springer Berlin Heidelberg. https://doi.org/10.1007/978-3-642-03983-6_26

[12] Sahu, P. K., Wu, E. H. K., \& Sahoo, J. "DuRT: Dual RSSI trend based localization for wireless sensor networks” IEEE Sensors Journal, 13(8), 3115-3123. https://doi.org/10.1109/JSEN.2013.2257731

[13] Garcia-Pineda, M., Tomás, J., Boronat, F. and Lloret, J. "The Development of Two Systems for Indoor Wireless Sensors Self-location”Ad Hoc \& Sensor Wireless Networks. 8. 235-258.

[14] Lloret, J., Tomás, J., Garcia-Pineda, M. and Canovas A. “A Hybrid Stochastic Approach for Self-Location of Wireless Sensors in Indoor Environments” Sensors, 2009, 9, 3695-3712 https://doi.org/10.3390/s90503695

[15] Fn-Link Tech. Ltd., nd. Realtek RTL8723BU Wi-Fi+BT Module Product Specification. [pdf],Available at: < http://www.fn-link.com/downloadRepository/113cd977-6c91-4f698b97-63fc138fa254.pdf> (Accessed: 20 September 2016).

[16] Liu, H., Darabi, H., Banerjee, P. and Liu, J. "Survey of wireless indoor positioning techniques and systems”, IEEE Transactions on Systems, Man and Cybernetics, Part C (Applications and Reviews), 37(6), pp. 1067-1080. https://doi.org/10.1109/ TSMCC.2007.905750.

[17] Gigl, T., Janssen, G.J.M., Dizdarevic, V., Witrisal, K. and Irahhauten, Z. “Analysis of a UWB indoor positioning system based on received signal strength”, 4th Workshop on Positioning, Navigation and Communication, . https://doi.org/10.1109/ WPNC.2007.353618

[18] Lau, E.-E. and Chung, W.-Y. "Enhanced RSSI-Based real-time user location tracking system for indoor and outdoor environments", 2007 International Conference on Convergence Information Technology (ICCIT 2007), · https://doi.org/10.1109/ ICCIT.2007.253

[19] ITU. "Propagation data and prediction methods for the planning of indoor radiocommunication systems and radio local area networks in the frequency range $300 \mathrm{MHz}$ to $100 \mathrm{GHz}$, P.1238-8 (07/2015)

[20] Bose, A., \& Foh, C. H. “A practical path loss model for indoor WiFi positioning enhancement” IEEE Information, Communications \& Signal Processing, 2007 6th 
International Conference (pp. 1-5).. 21-23 Nov. 2007, Gyeongju, South Korea https://doi.org/10.1109/ICCIT.2007.253

[21] Seybold, J.S.”Introduction to RF propagation” Chichester, United Kingdom: Wiley-Blackwell (an imprint of John Wiley \& Sons Ltd).

[22] Sommer, C. and Dressler, F. "Using the right two-ray model? A measurement based evaluation of PHY models in VANETs”, In: Proc. ACM MobiCom, 2011, pp. 1-3.

[23] Rappaport, T.S. “Wireless communications: Principles and practice” United States: IEEE Publications,U.S.

[24] Taoglas Antenna Solutions, "2.4 GHz Miniature Screw Mount Monopole Antenna Specification.” [pdf] Taoglas Antenna Solutions, www.taoglas.com. Available at: http://www.taoglas.com/images/product_images/original_images/GW.26.0111 2.4GHz Band Monopole SMA(M).pdf (Accessed: 20 September 2016). 